\title{
Modeling of Weak Anisotropic Anchoring of Nematic Liquid Crystals in the Landau-de Gennes Theory
}

\author{
Eero Willman, F. Aníbal Fernández, Member, IEEE, Richard James, and Sally E. Day, Member, IEEE
}

\begin{abstract}
The anisotropic anchoring effect of a treated solid surface on a nematic liquid crystal is described in the Landau-de Gennes theory using a power expansion on the tensor-order parameter and two mutually orthogonal unit vectors. The expression has three degrees of freedom, allowing for independent assignment of polar and azimuthal anchoring strengths and a preferred value of the surface-order parameter. It is shown that in the limit for a uniaxial constant-order parameter, the expression simplifies to the anisotropic generalization of the Rapini-Papoular anchoring energy density proposed by Zhao et al. Experimentally measurable values with a physical meaning in the Oseen-Frank theory can be scaled and assigned to the scalar coefficients of the tensor-orderparameter expansion. Results of numerical experiments comparing the anchoring according to the study of Zhao et al. in the Oseen-Frank theory and the power expansion in the Landau-de Gennes theory are presented and shown to agree well.
\end{abstract}

Index Terms-Anchoring, liquid crystal (LC).

\section{INTRODUCTION}

$\mathbf{T}$ HE OSEEN-FRANK continuum theory [1], [2] has been successfully used in the past to explain the elastic behavior of nematic liquid crystals (LCs). The requirement of new devices of high-resolution and lower energy consumption implies small dimensions and complex structures where topological defects are more likely to occur and even more, to play an important role in the operation of the device. In this case, the Oseen-Frank theory, which assumes the orientational order, remains constant throughout the cell, can no longer be used to model accurately the operation of the device. Instead, the Landau-de Gennes theory [3], which allows variation of the order parameter and biaxiality, provides a more accurate description of structures containing defects.

The operation of LC devices relies on the aligning effect of anchoring the LC to the solid surfaces of the cells. This effect can be achieved treating the surfaces by a number of means, including rubbing a polymer coating, photoalignment, or oblique evaporation techniques (for a review, see, e.g., [4]). The physical/chemical processes behind the anchoring and aligning effect of the surface include complex molecular interactions between the LC material and the treated surface. Electric fields due to a finite surface charge density and polarization due to

Manuscript received March 27, 2007; revised June 20, 2007.The review of this paper was arranged by Editor H.-S. Tae.

The authors are with the Department of Electronic and Electrical Engineering, University College London, WC1E 7JE London, U.K. (e-mail: e.willman@ee.ucl.ac.uk).

Digital Object Identifier 10.1109/TED.2007.904369 gradients in the nematic order and the director contribute to the alignment [3], [5]-[8]. This means that from a macroscopic point of view, it is impractical to try to model directly the treated surface itself. Instead, a phenomenological approach describing the effect the surface has on the LC is more useful in device modeling.

In both the Oseen-Frank and Landau-de Gennes continuum models, the anchoring is described by additional surface-energy terms. In the case of strong anchoring, the anchoring energy is very large, and the LC can be considered simply fixed at the boundary. Sometimes, however, this assumption is inadequate, as in the case of weak anchoring, where the LC material is no longer rigidly fixed to the surface. The expression for the anchoring energy is some finite function that depends of the LC properties at the surface and the surface properties. A minimum in this energy expression corresponds to the "easy" or preferred state of the anchoring. In the Oseen-Frank theory, the parameters involved are the anchoring strength and the easy direction only, while in the Landau-de Gennes theory, a preferred distribution of nematic order at the surface should also be taken into account.

In the modeling of LC devices, it has been common practice to make the simplifying assumption (Rapini-Papoular (RP) [9]) that the anchoring energy density varies in a $W \sin ^{2}(\Theta)$ fashion with respect to the easy direction, where $W$ is an anchoring strength coefficient and $\Theta$ is the angle between the director and the easy direction, irrespective of the relative position of the anchoring surface. However, the surface anchoring is usually anisotropic; more specifically, the anchoring tends to be stronger in the polar (departing from the surface) rather than in the azimuthal direction (on the surface) [10]. The anchoring strengths depend both on the applied surface treatment and the LC material used. Reported polar anchoring strengths typically lie in the range from $10^{-7}$ to $10^{-3} \mathrm{~J} / \mathrm{m}^{2}$, whereas azimuthal anchoring strengths are typically one or two orders of magnitude smaller [11]-[14]. For this reason, various generalizations that take into account the difference between polar and azimuthal anchoring strengths have been introduced in the Oseen-Frank theory by several authors [15]-[18].

The Landau-de Gennes theory has been extensively used to explain various aspects of the fundamental physics of LCs, including the solid surface-LC interface. Anchoring transitions and orientational wetting at the interface has been examined theoretically using various expressions to describe the effect of the solid surface has on the LC material [19]-[22]. However, 
the inclusion of anisotropic weak anchoring characterized by experimentally measurable parameters into a numerical model has not received much attention within this framework. In what follows, we attempt to address this issue.

A brief review of the relevant anchoring expressions both in the Oseen-Frank and Landau-de Gennes theories is presented in Section II. Section III introduces a power expansion of the tensor-order parameter $\mathbf{Q}$ to describe the anisotropic anchoring of a treated solid surface in the Landau-de Gennes theory. Finally, in Section IV, results of numerical simulations comparing weak anchoring in the Oseen-Frank and Landau-de Gennes theories are shown.

\section{REVIEW OF EXISTING WEAK ANCHORING REPRESENTATIONS}

\section{A. Anchoring in the Oseen-Frank Theory}

Probably the first and best known expression describing the weak anchoring effect in the Oseen-Frank theory is the RP expression [9]. This assumes that the anchoring energy density increases in $\sin ^{2}$ fashion as the director deviates from the easy direction

$$
F_{\mathrm{RP}}=W \sin ^{2}(\Theta)
$$

where $W$ is a scalar value known as the anchoring strength, and $\Theta$ is the angle of departure of the director $\mathbf{n}$ from the easy direction ê.

The main weakness of (1) is its inability to distinguish between different directions of angular departures from ê. This means that the difference between polar and azimuthal anchoring strengths cannot be taken into account. Despite this, the RP anchoring is a widely used approximation and often used as a reference to which other anchoring representations are compared. The $\sin ^{2}$ variation in energy is also an assumption often made when experimentally measuring the surface anchoring strength coefficients, for example using the torque balance [23] or the improved torque balance methods [24].

A generalization of the RP expression that differentiates between polar and azimuthal anchoring strengths is (e.g., [17])

$$
F_{\mathrm{RPgen}}=A_{1} \sin ^{2}\left(\theta-\theta_{e}\right)+A_{2} \sin ^{2}\left(\phi-\phi_{e}\right)
$$

where $A_{1}$ and $A_{2}$ refer to polar and azimuthal anchoring strengths and $\theta, \phi, \theta_{e}$, and $\phi_{e}$ refer to the tilt and azimuthal angles of the director and easy direction, respectively. However, this approach completely decouples the two angles in an unrealistic way giving rise to complications. First, the decoupling of the two angles makes the anchoring energy density discontinuous with respect to $\theta$ and $\phi$ [16]. Second, the azimuthal anchoring energy density should also depend on the tilt angle of the director. Furthermore, (2) is periodic with a period of $\pi$ radians, resulting in a bistable anchoring when the tilt angle of the easy direction lies in the range $0<\theta_{e}<\pi / 2$.

It has later been shown by Zhao et al. [15], [16] that a representation of the anisotropic surface-energy density without the complications outlined above is

$$
F_{\mathrm{ZWI}}=B_{1} \sin ^{2}(\Theta) \cos ^{2}\left(\Phi-\Psi_{0}\right)+B_{2} \sin ^{2}(\Theta) \sin ^{2}\left(\Phi-\Psi_{0}\right)
$$

where $(\Theta, \Phi)$ are angular deviations of the director from ê in a local coordinate system defined by the orthonormal vector triplet $\left(\hat{\mathbf{v}}_{1}, \hat{\mathbf{v}}_{2}, \hat{\mathbf{e}}\right)$ describing the principal axes of anchoring. Equation (3) can also be expressed more compactly as [15]

$$
F_{\mathrm{ZWI}}=B_{1}\left(\hat{\mathbf{v}}_{1} \cdot \mathbf{n}\right)^{2}+B_{2}\left(\hat{\mathbf{v}}_{2} \cdot \mathbf{n}\right)^{2}
$$

where $B_{1}$ and $B_{2}$ are the anchoring strength coefficients corresponding to deformations in the $\left(\hat{\mathbf{v}}_{1}, \hat{\mathbf{e}}\right)$ and $\left(\hat{\mathbf{v}}_{2}, \hat{\mathbf{e}}\right)$ planes, respectively. It can be shown by comparing equal order terms of spherical harmonics expansions of (2) and (3) that the anchoring strength coefficients in the two expressions can be related by

$$
\begin{aligned}
B_{1} & =\frac{1}{2}\left(A_{1}-\frac{5}{8} A_{2}\right) \\
B_{2} & =\frac{1}{2}\left(A_{1}+\frac{5}{8} A_{2}\right) .
\end{aligned}
$$

\section{B. Anchoring in the Landau-de Gennes Theory}

In the Landau-de Gennes theory, a simple way of approximating the anchoring effect of a treated or rubbed surface is by means of a penalty-type expression [10], [21]

$$
F_{\text {pen }}=W\left(\mathbf{Q}-\mathbf{Q}_{e}\right)^{2} .
$$

In (6), $\mathbf{Q}_{e}$ is the preferred, easy $\mathbf{Q}$. Clearly, the energy density is minimized when $\mathbf{Q}=\mathbf{Q}_{e}$. Equation (6) shows a $\sin ^{2}$ variation with respect to angular departures from the easy direction and a quadratic variation with respect to the easy surface-order parameter. However, similarly to (1), the anchoring represented by (6) does not distinguish between polar and azimuthal anchoring strengths.

Another expression for the surface-energy density in the Landau-de Gennes theory describes the effect of an isotropic surface on the LC material, i.e., a surface giving degenerate alignment, where only the director tilt is constrained. This is a Landau power series expansion on the surface normal unit vector $\hat{\mathbf{v}}$ and $\mathbf{Q}[20]$

$$
F_{\exp }=c_{1}(\hat{\mathbf{v}} \cdot \mathbf{Q} \cdot \hat{\mathbf{v}})+c_{2} \operatorname{Tr}\left(\mathbf{Q}^{2}\right)+c_{3}(\hat{\mathbf{v}} \cdot \mathbf{Q} \cdot \hat{\mathbf{v}})^{2}+c_{4}\left(\hat{\mathbf{v}} \cdot \mathbf{Q}^{2} \cdot \hat{\mathbf{v}}\right) .
$$

Here, $c_{i}$ are scalar coefficients that determine the preferred tilt angle and surface order. Equation (7) has been used in, e.g., [25] and [26] to study anchoring transitions.

An expression for anisotropic anchoring, linear in $\mathbf{Q}$, has been studied in [22]

$$
F_{\text {lin }}=-\operatorname{Tr}(\mathbf{h} \cdot \mathbf{Q})
$$

where $\mathbf{h}$ is a symmetric traceless tensor describing the symmetry of the surface. Since this expression is linear, there is no control over the surface-order parameter which tends to either positive or negative infinity depending on $\mathbf{h}$. 


\section{ANChoring ENERgy Density of AN ANISOTROPIC SURFACE IN THE LANDAU-DE GENNES THEORY}

A generalization with a reduction in symmetry as compared to (7) can be written as a power expansion, truncated to second order, on the $Q$-tensor and two orthogonal unit vectors whose directions are determined by the surface treatment

$$
\begin{aligned}
F_{s}= & a \operatorname{Tr}\left(\mathbf{Q}^{2}\right)+W_{1}\left(\hat{\mathbf{v}}_{1} \cdot \mathbf{Q} \cdot \hat{\mathbf{v}}_{1}\right)+W_{2}\left(\hat{\mathbf{v}}_{2} \cdot \mathbf{Q} \cdot \hat{\mathbf{v}}_{2}\right) \\
& +W_{3}\left(\hat{\mathbf{v}}_{1} \cdot \mathbf{Q} \cdot \hat{\mathbf{v}}_{2}\right)+X_{1}\left(\hat{\mathbf{v}}_{1} \cdot \mathbf{Q} \cdot \hat{\mathbf{v}}_{1}\right)^{2} \\
& +X_{2}\left(\hat{\mathbf{v}}_{2} \cdot \mathbf{Q} \cdot \hat{\mathbf{v}}_{2}\right)^{2}+X_{3}\left(\hat{\mathbf{v}}_{1} \cdot \mathbf{Q} \cdot \hat{\mathbf{v}}_{2}\right)^{2} \\
& +X_{4}\left(\hat{\mathbf{v}}_{1} \cdot \mathbf{Q}^{2} \cdot \hat{\mathbf{v}}_{1}\right)+X_{5}\left(\hat{\mathbf{v}}_{2} \cdot \mathbf{Q}^{2} \cdot \hat{\mathbf{v}}_{2}\right) \\
& +X_{6}\left(\hat{\mathbf{v}}_{1} \cdot \mathbf{Q}^{2} \cdot \hat{\mathbf{v}}_{2}\right)+X_{7}\left(\hat{\mathbf{v}}_{1} \cdot \mathbf{Q} \cdot \hat{\mathbf{v}}_{1}\right)\left(\hat{\mathbf{v}}_{2} \cdot \mathbf{Q} \cdot \hat{\mathbf{v}}_{2}\right) \\
& +X_{8}\left(\hat{\mathbf{v}}_{1} \cdot \mathbf{Q} \cdot \hat{\mathbf{v}}_{2}\right)\left(\hat{\mathbf{v}}_{1} \cdot \mathbf{Q} \cdot \hat{\mathbf{v}}_{2}\right) \\
& +X_{9}\left(\hat{\mathbf{v}}_{1} \cdot \mathbf{Q} \cdot \hat{\mathbf{v}}_{2}\right)\left(\hat{\mathbf{v}}_{1} \cdot \mathbf{Q} \cdot \hat{\mathbf{v}}_{1}\right) \\
& +X_{10}\left(\hat{\mathbf{v}}_{1} \cdot \mathbf{Q} \cdot \hat{\mathbf{v}}_{2}\right)\left(\hat{\mathbf{v}}_{2} \cdot \mathbf{Q} \cdot \hat{\mathbf{v}}_{2}\right)
\end{aligned}
$$

where $W_{i}$ and $X_{i}$ are the anchoring strength coefficients. The simplest case that still allows for anisotropic anchoring with a preferred order parameter is when the scalar coefficients $W_{3}$ and $X_{i}$ are zero. In this case, the surface anchoring energy reduces to

$$
F_{s}=a \operatorname{Tr}\left(\mathbf{Q}^{2}\right)+W_{1}\left(\hat{\mathbf{v}}_{1} \cdot \mathbf{Q} \cdot \hat{\mathbf{v}}_{1}\right)+W_{2}\left(\hat{\mathbf{v}}_{2} \cdot \mathbf{Q} \cdot \hat{\mathbf{v}}_{2}\right) .
$$

The principal axes of anchoring $\left(\hat{\mathbf{e}}, \hat{\mathbf{v}}_{1}, \hat{\mathbf{v}}_{2}\right)$ are the easy direction and two mutually orthogonal unit vectors, respectively, so that $\hat{\mathbf{e}}=\hat{\mathbf{v}}_{1} \times \hat{\mathbf{v}}_{2}$. Equation (10) can be directly discretized for implementation, but is here expanded in an analytical form in order to show how meaningful values can be assigned to the scalar coefficients $a, W_{1}$, and $W_{2}$. $\hat{\mathbf{e}}$ is the easy direction only when both $W_{1}$ and $W_{2}$ are positive scalars. If $W_{i}=0$ and $W_{j}>0$, the anchoring becomes degenerate in the $\left(\hat{\mathbf{e}}, \hat{\mathbf{v}}_{i}\right)$ plane. Setting $W_{1}$ or $W_{2}$ to a negative value minimizes $F_{s}$ in the direction of $\hat{\mathbf{v}}_{1}$ or $\hat{\mathbf{v}}_{2}$, and $\hat{\mathbf{e}}$ loses its physical meaning as the easy direction. The types of anchoring achieved by using negative coefficients are equivalent to a rotation of the principal axes when using positive $W_{1}$ and $W_{2}$. For this reason, only cases of nonnegative anchoring strength coefficients are considered in what follows.

Without loss of generality, the geometry can be defined locally: $\left(\hat{\mathbf{e}}, \hat{\mathbf{v}}_{1}, \hat{\mathbf{v}}_{2}\right)$ are chosen to coincide with the $(x, y, z)$ coordinates. The traceless $\mathbf{Q}$ is defined as

$$
Q_{i j}=\frac{S}{2}\left(3 n_{i} n_{j}-\delta_{i j}\right)+P\left(k_{i} k_{j}-l_{i} l_{j}\right)
$$

where $S$ is the scalar order parameter and $P$ is the biaxiality parameter. Numerically, $S$ is the dominant eigenvalue of $\mathbf{Q}$, whereas $P$ is half the difference between the two other eigenvalues. $\mathbf{n}, \mathbf{k}$, and $\mathbf{l}$, are the director and two vectors that define the direction of nematic order in three dimensions, and $\delta_{i j}$ is the Kronecker delta.

The three orthogonal unit vectors $\mathbf{n}, \mathbf{k}$, and $\mathbf{l}$ can be written in terms of the three angles $\alpha, \beta$, and $\gamma$, where $\alpha$ is the angular deviation of $\mathbf{n}$ from the ( $\left.\hat{\mathbf{e}}, \hat{\mathbf{v}}_{2}\right)$ plane (local twist), $\beta$ is the angular deviation of $\mathbf{n}$ from the ( $\left.\hat{\mathbf{e}}, \hat{\mathbf{v}}_{1}\right)$ plane (local tilt), and $\gamma$ is a rotation of $\mathbf{k}$ and $\mathbf{l}$ around $\mathbf{n}$ determining the orientation of the plane of biaxial order

$$
\begin{aligned}
& \mathbf{n}=\left(\begin{array}{c}
\cos (\alpha) \cos (\beta) \\
-\sin (\alpha) \cos (\beta) \\
\sin (\beta)
\end{array}\right) \\
& \mathbf{k}=\left(\begin{array}{c}
\sin (\alpha) \cos (\gamma)+\cos (\alpha) \sin (\beta) \sin (\gamma) \\
\cos (\alpha) \cos (\gamma)-\sin (\alpha) \sin (\beta) \sin (\gamma) \\
-\cos (\beta) \sin (\gamma)
\end{array}\right) \\
& \mathbf{l}=\left(\begin{array}{c}
\sin (\alpha) \sin (\gamma)-\cos (\alpha) \sin (\beta) \cos (\gamma) \\
\cos (\alpha) \sin (\gamma)+\sin (\alpha) \sin (\beta) \cos (\gamma) \\
\cos (\beta) \cos (\gamma)
\end{array}\right)
\end{aligned}
$$

so that when $\alpha=\beta=\gamma=0,(\mathbf{n}, \mathbf{k}, \mathbf{l})=\left(\hat{\mathbf{e}}, \hat{\mathbf{v}}_{1}, \hat{\mathbf{v}}_{2}\right)$. Equation (10) can then be written in terms of $S, P, \alpha, \beta$, and $\gamma$ as

$$
\begin{aligned}
F_{s}= & a\left(\frac{3}{2} S^{2}+2 P^{2}\right) \\
& +W_{1}\left\{F_{1 S}(S, \alpha, \beta)+F_{1 P}(P, \alpha, \beta, \gamma)\right\} \\
& +W_{2}\left\{F_{2 S}(S, \beta)+F_{2 P}(P, \beta, \gamma)\right\}
\end{aligned}
$$

where $F_{1 S}$ and $F_{2 S}$ are

$$
\begin{aligned}
F_{1 S}(S, \alpha, \beta) & =\frac{S}{2}\left(3 \sin ^{2} \alpha \cos ^{2} \beta-1\right) \\
& =\frac{3 S}{2}\left(\mathbf{n} \cdot \hat{\mathbf{v}}_{1}\right)^{2}-\frac{S}{2}
\end{aligned}
$$

and

$$
\begin{aligned}
F_{2 S}(S, \beta) & =\frac{S}{2}\left(3 \sin ^{2} \beta-1\right) \\
& =\frac{3 S}{2}\left(\mathbf{n} \cdot \hat{\mathbf{v}}_{2}\right)^{2}-\frac{S}{2} .
\end{aligned}
$$

In the limit of constant uniaxial order $F_{1 P}, F_{2 P}$ and the isotropic part of $F_{s}$ can be ignored, and (10) reduces to the sum of (16) and (17) multiplied by $W_{1}$ and $W_{2}$, respectively. In this case, (10) is equivalent to (4) of Zhao et al., with anchoring strength coefficients related by a factor of $3 S / 2$. Fig. 1 shows the angular variation of the anchoring energy density for different values of the polar to azimuthal anchoring ratio, $R=W_{2} / W_{1}$ when order parameter variations are not considered.

Without the simplification of constant uniaxial order, the preferred surface-order and biaxiality parameters $S_{e}$ and $P_{e}$ that minimize (10) are determined by the relative values of $W_{1}, W_{2}$, and $a$. The two constants $W_{1}$ and $W_{2}$ define the anisotropic azimuthal and polar anchoring strengths, and the value of $a$ determines the resulting easy surface order. The preferred surface order occurs when $\mathbf{n}=\hat{\mathbf{e}}$, i.e., $\alpha=\beta=0$. Equation (15) then simplifies to

$$
\begin{array}{r}
F_{s}=a\left(\frac{3}{2} S^{2}+2 P^{2}\right)+W_{1}\left\{\left(2 \cos ^{2} \gamma-1\right) P-\frac{1}{2} S\right\} \\
+W_{2}\left\{\left(-2 \cos ^{2} \gamma+1\right) P-\frac{1}{2} S\right\} .
\end{array}
$$




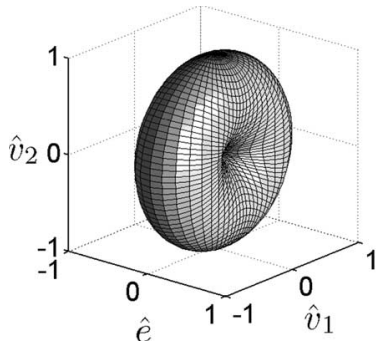

(a)

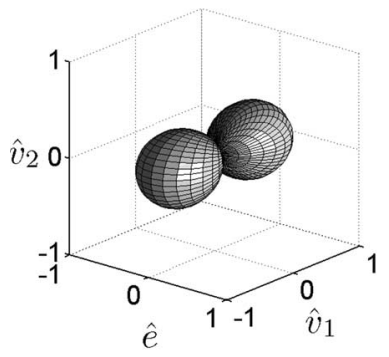

(c)

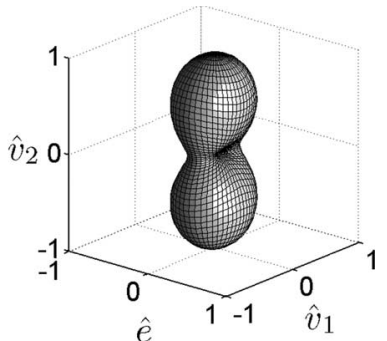

(b)

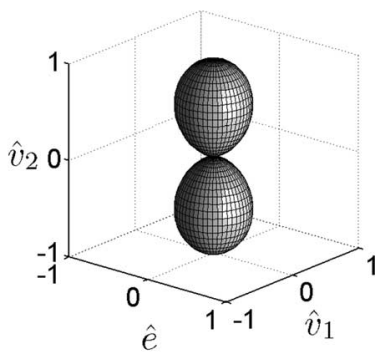

(d)
Fig. 1. Normalized anisotropic parts of the anchoring energy density for a surface with $\hat{\mathbf{e}}=[1,0,0], \hat{\mathbf{v}}_{1}=[0,1,0]$, and $\hat{\mathbf{v}}_{2}=[0,0,1]$. (a) $R=1$. (b) $R=3$. (c) $R=0$. (d) $R=\infty$. $\left(R=W_{2} / W_{1}\right)$.

The value of $a$ which minimizes $F_{s}$ for a given value of the surface-order parameter $S_{e}$ can be found by minimizing (18) with respect to $S$, giving

$$
a=\frac{W_{1}+W_{2}}{6 S_{e}} .
$$

The resulting biaxiality parameter distribution as function of $\gamma$ in the plane of $\mathbf{l}$ and $\mathbf{k}$ is found in a similar fashion by minimizing (18) with respect to $P$ and substituting $a$ from (19) giving

$$
P_{e}=\frac{1-R}{1+R}\left\{1-2 \cos ^{2} \gamma\right\} \frac{3}{2} S_{e} .
$$

Alternatively, in terms of the eigenvalues of $\mathbf{Q},(10)$ is minimized when the eigenvalue in the direction of $\hat{\mathbf{e}}$ is $\lambda_{\hat{\mathbf{e}}}=S_{e}$, and the difference between the two remaining eigenvalues is $\lambda_{\hat{\mathbf{v}}_{1}}-\lambda_{\hat{\mathbf{v}}_{2}}=2 P_{e}$. Fig. 2 shows the three eigenvalues of the $\mathbf{Q}$ that minimizes the surface-energy density of (10) as a function of $R$, normalized for $S_{e}=1$. Two cases can be identified from the figure.

1) $R=1$ : The two anchoring strength coefficients are equal, $\left(W_{1}=W_{2}\right)$ and $\lambda_{\hat{\mathbf{v}}_{1}}=\lambda_{\hat{\mathbf{v}}_{2}}=-\lambda_{\hat{\mathbf{e}}} / 2$, so that $\mathbf{Q}$ at the surface is uniaxial with a positive order parameter $S=\lambda_{\hat{\mathbf{e}}}=S_{e}$ and $\mathbf{n}=\hat{\mathbf{e}}$.

2) $R<1$ or $R>1$ : The two anchoring strength coefficients are not equal. As $R$ varies from 1 to 0 or from 1 to $\infty$, the surface order undergoes a transition from a positive uniaxial order to a negative uniaxial order through a biaxial state. In the limits of $R=0$ or $R=\infty$, when either $W_{1}$ or $W_{2}$ is zero, the anchoring is planar degenerate with a uniaxial negative scalar order parameter of value $S=$ $-2 S_{e}$, with $\mathbf{n}$ parallel to the unit vector corresponding to the nonzero anchoring coefficient.

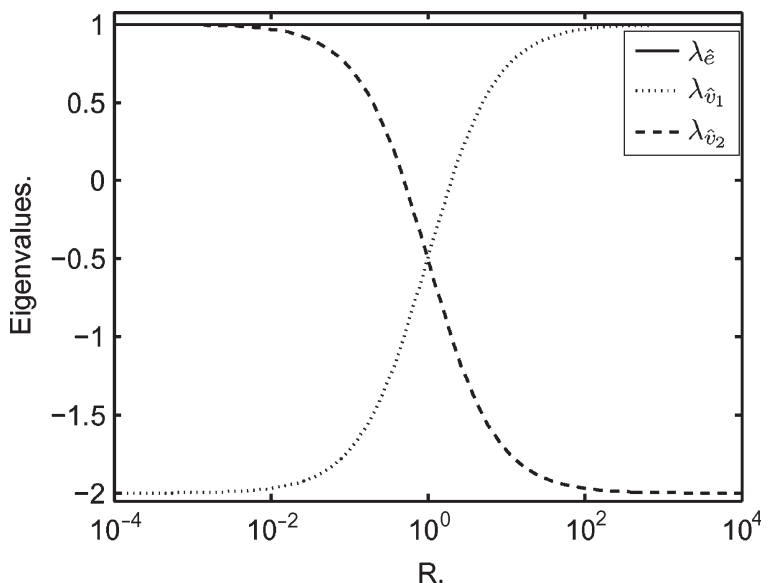

Fig. 2. Eigenvalues of a $\mathbf{Q}$ that minimizes the surface-energy density as a function of $R$, when $S_{e}$ is unity.

However, a more complete description of the surface order needs to include the bulk energy density terms, which in the standard Landau-de Gennes theory for nematic LCs favor a uniaxial $\mathbf{Q}$ with a positive scalar order parameter $S=S_{0}$ [3]. The resulting surface $\mathbf{Q}$ then describes a state that minimizes the combination of the surface and bulk terms.

Figs. 3-5 show the calculated variation in order for various anchoring conditions when the bulk thermotropic coefficients for the 5CB LC [27] are used with the single elastic coefficient approximation and $K=5 \mathrm{pN}$. When the anchoring energy is low, the bulk terms dominate and $\mathbf{Q}$ at the surface is close to the bulk equilibrium value for all $R$. Figs. 3 and 4 show the variation of the order parameter $\left(S=\lambda_{e}\right)$ and biaxiality parameter $\left(P=\left(\lambda_{\hat{\mathbf{v}}_{1}}-\lambda_{\hat{\mathbf{v}}_{2}}\right) / 2\right)$ with $R$ and the distance to the surface when $W_{2} \approx 5 \times 10^{-5} \mathrm{~J} / \mathrm{m}^{2}$. A small degree of biaxial order is induced at the surface when $R>1$, resulting in a decrease in $S$. The variations in order are contained within about a 10-nm-thick transition region near the surface. Fig. 5 shows the eigenvalues of $\mathbf{Q}$ at the surface, normalized by $S_{0}$, as functions of $W_{2}$ for $R=1,3$, and $\infty$. For comparison, the eigenvalues corresponding to a linear surface-energy density ( $a=0$ ) when $R=1$ are also shown (marked with circles). The influence of increased anchoring strengths can be observed in the eigenvalues. The surface energy becomes comparable to the bulk energy in the region around $W_{2}=10^{-3}$ to $10^{-1} \mathrm{~J} / \mathrm{m}^{2}$, where a reduction in $\lambda_{e}$ can be observed. As $W_{2}$ is further increased, the surface anchoring becomes the dominant energy term, and the eigenvalues converge toward those that minimize the surface energy, as shown in Fig. 2.

\section{NUMERICAL RESULTS}

Results of numerical simulations using the weak anchoring equation of (10) are presented next. First, results of simulations of the switching of a twisted nematic cell using the Landau-de Gennes and the Oseen-Frank theories with weak anchoring are shown. Then, the effect of anchoring induced biaxiality on the effective anchoring strength is investigated in the Landau-de Gennes theory. 


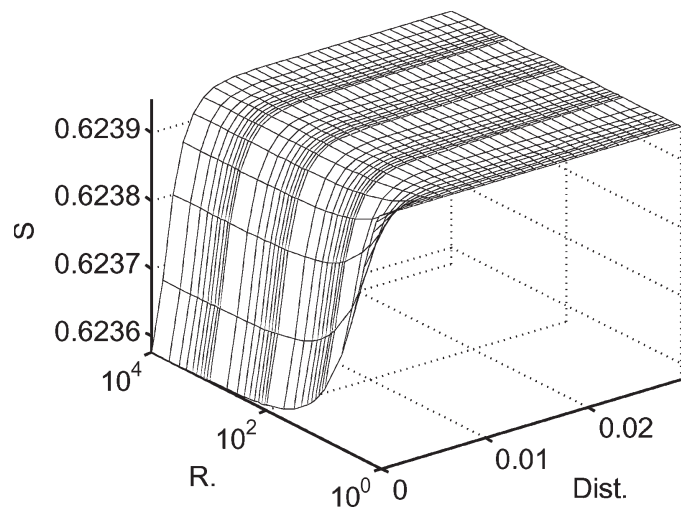

Fig. 3. Scalar order parameter $S$ as a function of the distance from the surface (in micrometers) and the ratio $R$ between $W_{2}$ and $W_{1}$.

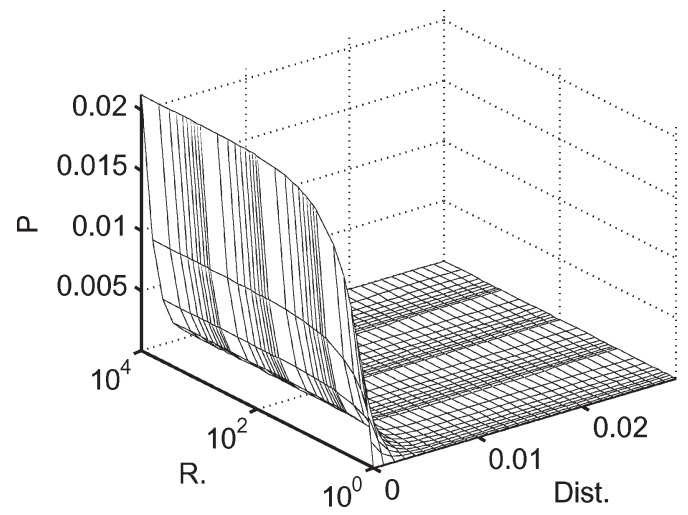

Fig. 4. Biaxiality parameter $P$ as a function of the distance from the surface (in micrometers) and the ratio $R$ between $W_{2}$ and $W_{1}$.

The numerical simulations are performed using finite element discretizations of the Landau-de Gennes and OseenFrank energies [28], [29], where the weak surface anchoring energy densities are modeled by (10) and (4), respectively. The two corresponding energy functionals are

$$
\begin{aligned}
F_{\mathrm{LdG}}=\int_{v}\{ & \frac{1}{2} A\left(T-T^{*}\right) \operatorname{Tr}\left(\mathbf{Q}^{2}\right)-\frac{1}{3} B \operatorname{Tr}\left(\mathbf{Q}^{3}\right) \\
& +\frac{1}{4} C \operatorname{Tr}\left(\mathbf{Q}^{2}\right)^{2}+\frac{1}{2} L_{1} Q_{\alpha \beta, \gamma} Q_{\alpha \beta, \gamma} \\
& +\frac{1}{2} L_{2} Q_{\alpha \beta, \beta} Q_{\alpha \gamma, \gamma}+\frac{1}{2} L_{3} Q_{\alpha \beta} Q_{\gamma \mu, \alpha} Q_{\gamma \mu, \beta} \\
& \left.-\frac{1}{2} \varepsilon_{0}(\mathbf{E} \cdot \overline{\bar{\varepsilon}} \cdot \mathbf{E})\right\} d v+\int_{s} F_{s} d s
\end{aligned}
$$

and

$$
\begin{array}{r}
F_{\mathrm{OF}}=\int_{v}\left\{\frac{K_{11}}{2}(\nabla \cdot \mathbf{n})^{2}+\frac{K_{22}}{2}(\mathbf{n} \cdot \nabla \times \mathbf{n})^{2}+\frac{K_{33}}{2}|\mathbf{n} \times \nabla \times \mathbf{n}|^{2}\right. \\
\left.-\frac{1}{2} \varepsilon_{0}(\mathbf{E} \cdot \overline{\bar{\varepsilon}} \cdot \mathbf{E})\right\} d v+\int_{s} F_{\mathrm{ZWI}} d s
\end{array}
$$

where $A, B$, and $C$ are bulk material thermotropic coefficients, $\overline{\bar{\varepsilon}}$ is the dielectric tensor, and $\mathbf{E}$ is the externally applied electric field. $L_{1}, L_{2}$, and $L_{3}$ are bulk elastic constants, related to

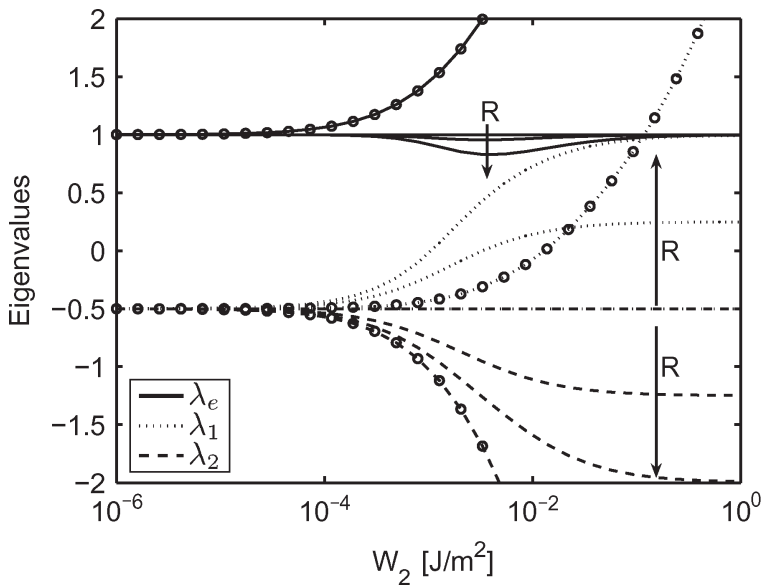

Fig. 5. Normalized eigenvalues of $\mathbf{Q}$ at the surface as a function of $W_{2}$ for $R=1,3$, and $\infty$, when $a$ is set according to expression 20 (no markers) and for the linear case $a=0$ and $R=1$ (circles).

the splay, twist, and bend coefficients $K_{11}, K_{22}$, and $K_{33}$ by [30], [31]

$$
\begin{aligned}
L_{1} & =\frac{2}{27 S_{0}^{2}}\left(K_{33}-K_{11}+3 K_{22}\right) \\
L_{2} & =\frac{4}{9 S_{0}^{2}}\left(K_{11}-K_{22}\right) \\
L_{3} & =\frac{4}{27 S_{0}^{3}}\left(K_{33}-K_{11}\right) .
\end{aligned}
$$

The values of the material parameters used are for $5 \mathrm{CB}$, with $\left(T-T^{*}\right)=-4^{\circ}$ giving an equilibrium order parameter $S_{0} \approx 0.624$ [27], [32], [33]

$$
\begin{aligned}
K_{11} & =6.2 \mathrm{pN} \\
K_{22} & =3.9 \mathrm{pN} \\
K_{33} & =8.2 \mathrm{pN} \\
A & =0.867 \times 10^{5} \mathrm{~N} \cdot \mathrm{m}^{-2} \cdot \mathrm{K}^{-1} \\
B & =2.133 \times 10^{6} \mathrm{~N} \cdot \mathrm{m}^{-2} \\
C & =1.733 \times 10^{6} \mathrm{~N} \cdot \mathrm{m}^{-2} \\
\Delta \varepsilon & =11.5 \\
\epsilon_{\|} & =7.0 .
\end{aligned}
$$

The polar anchoring strength coefficient $B_{2}$ of (4) is kept constant at $8 \times 10^{-4}$, and the azimuthal anchoring strength coefficient $B_{1}$ is set according to $B_{1}=B_{2} / R$ (Fig. 6). The anchoring strength coefficients of (10) are related to those of (4) by $W_{i}=2 B_{i} /\left(3 S_{0}\right)$. The isotropic surface-energy density coefficient $a$ is set using (19) so that $S_{e}=S_{0}$.

\section{A. Comparison Between the Landau-de Gennes and Oseen-Frank Models}

Using the procedure described above, the switching of a $1-\mu \mathrm{m}$-thick cell with $90^{\circ}$ twist between the aligning surfaces and $5^{\circ}$ pretilt on both surfaces were modeled using both theories. First, the tilt and twist angles at the surfaces and at the mid plane of the cell were recorded while keeping the ratio of the 


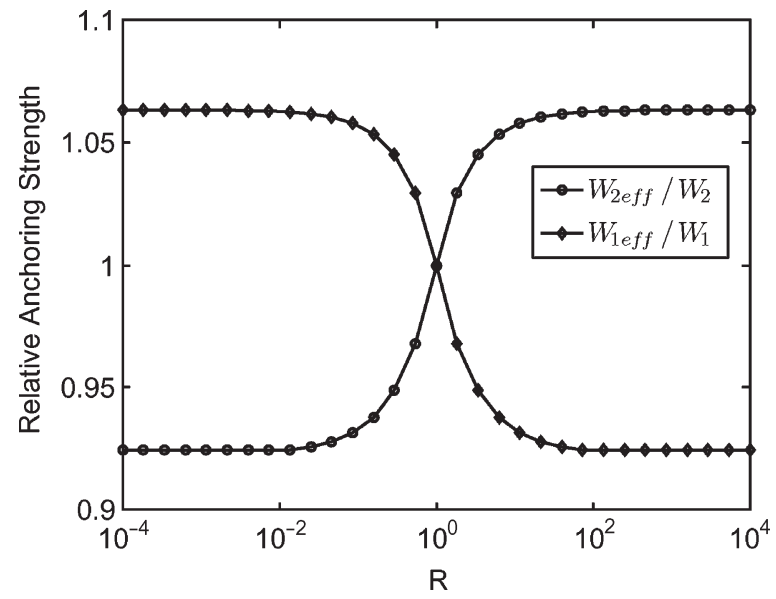

Fig. 6. Ratio of the effective azimuthal anchoring strength coefficient and $W_{1}$ as a function of $R$.

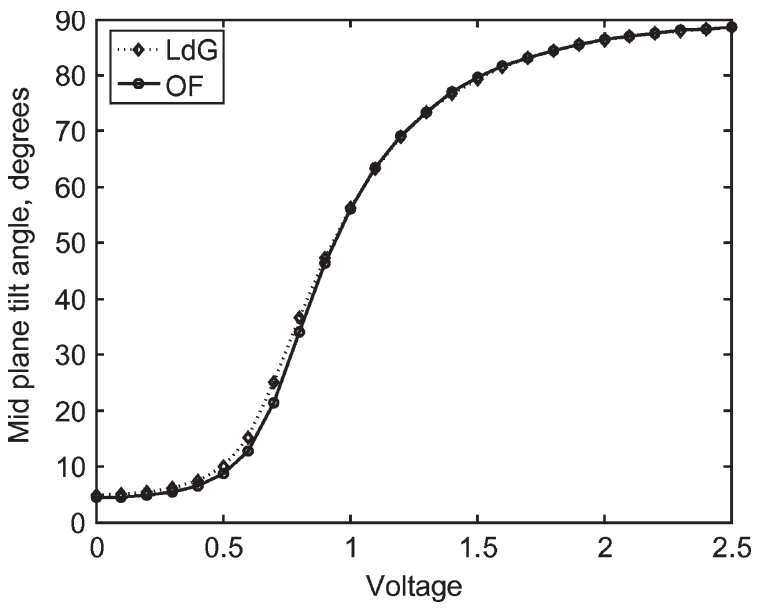

Fig. 7. Mid-plane tilt angles as a function of $V$.

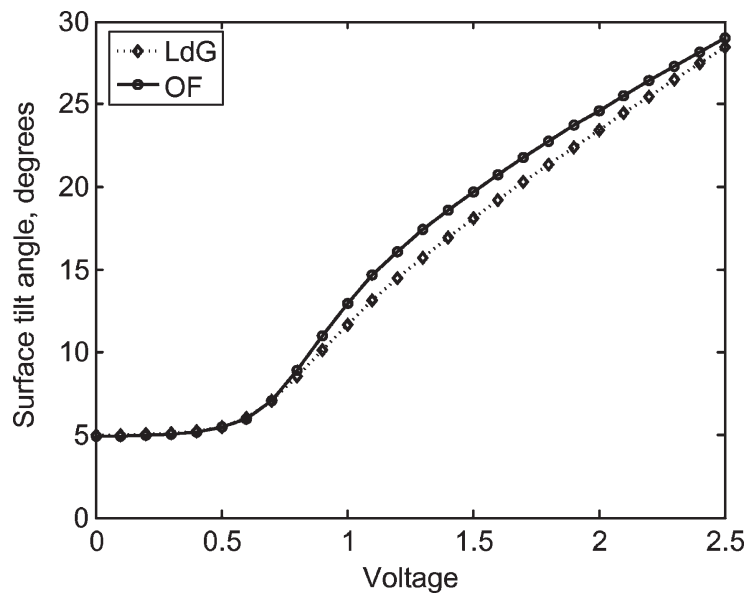

Fig. 8. Surface tilt angles as a function of $V$.

anchoring strengths constant at $R=3$, and the voltage applied across the cell was varied between 0 and $2.5 \mathrm{~V}$ (see Figs. 7-9). Then, the applied voltage was kept constant, but $R$ was varied in the range from 1 to $1 \times 10^{4}$, as shown in Figs. 10-12. The two simulations yield slightly different results, but this is to be expected since the Zhao et al. expression does not allow for order variations.

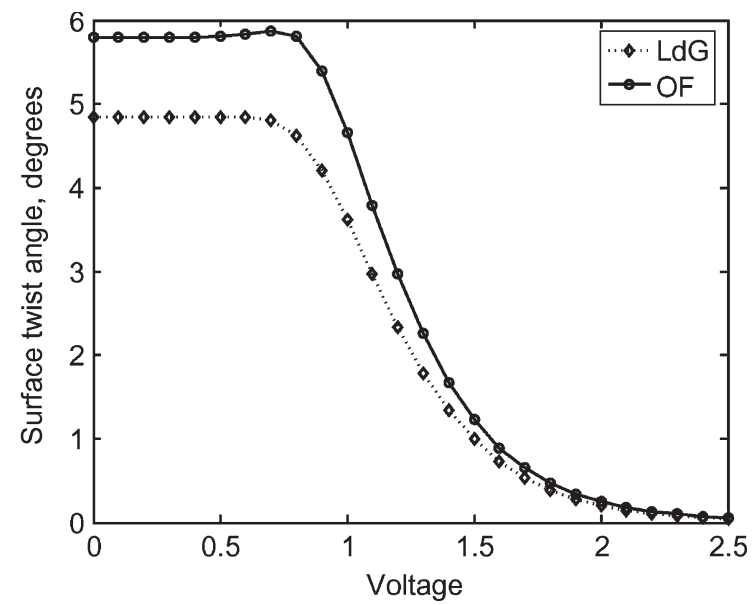

Fig. 9. Surface twist angles as a function of $V$.

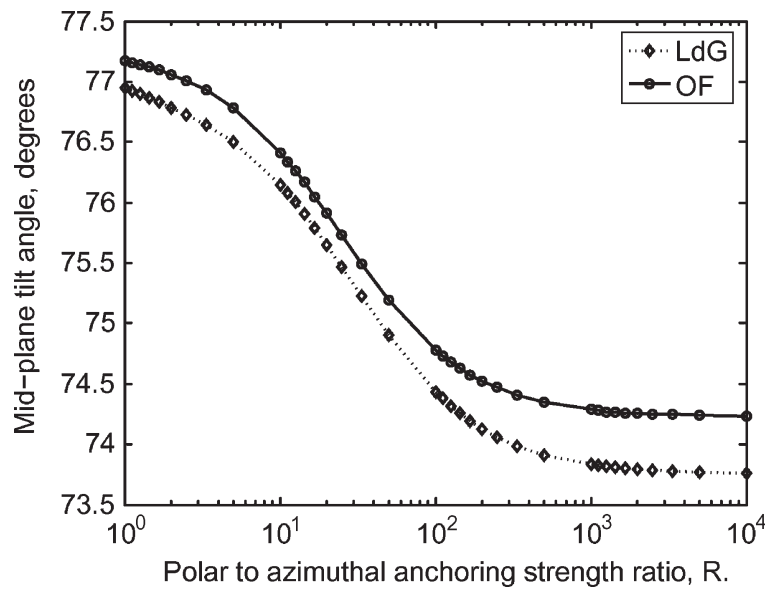

Fig. 10. Mid-plane tilt angles as a function of $R$.

\section{B. Effect of Order Variations on the Effective Anchoring Strength}

In Section III, a proportionality relationship with a factor of $3 S / 2$ between the anchoring strength coefficients $W_{i}$ of (10) and $B_{i}$ of (4) was established in the limit of constant uniaxial order. However, when $R \neq 1$, this assumption is not true implying that the anchoring energy density will be different from (4), and the actual effective anchoring strength $W_{\text {eff }}$ acting on the director will differ from the expected value of $W_{i}$ used in (10). In order to investigate this, the torque balance method [23], [24] is used in conjunction with modeling results of the $Q$-tensor distribution [28] to find $W_{\text {eff }}$ acting on the director.

In a twisted nematic cell without tilt, the distortion in the bulk produces an elastic torque that causes the director at the aligning surfaces to deviate from the easy direction by an angle $\Delta \phi$ that can be found experimentally [23]. Additionally, the anchoring at the surfaces cause elastic torques that tend to restore the director back to the easy direction. In the steady state, without externally applied fields, the elastic torques are equal but in opposing directions. In this case, it is possible to equate the elastic bulk twist energy to the surface anchoring energy (see, e.g., [23]). Using the Oseen-Frank theory, and 


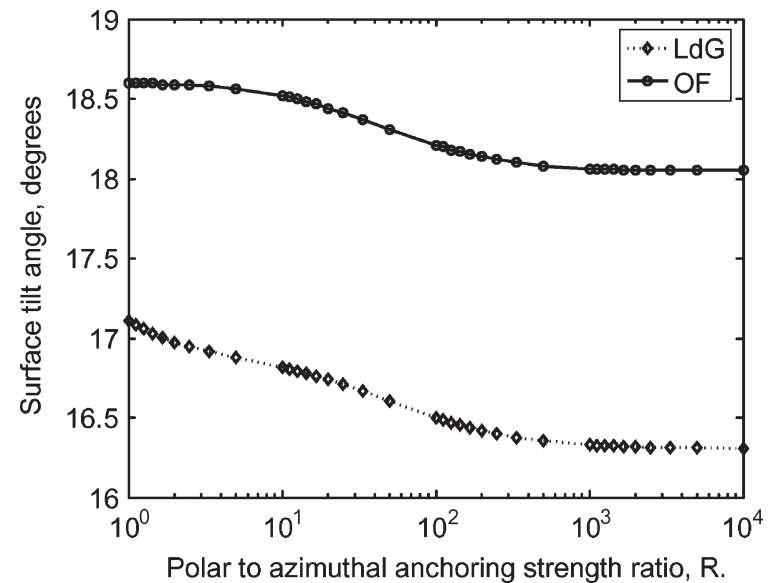

Fig. 11. Surface tilt angles as a function of $R$.

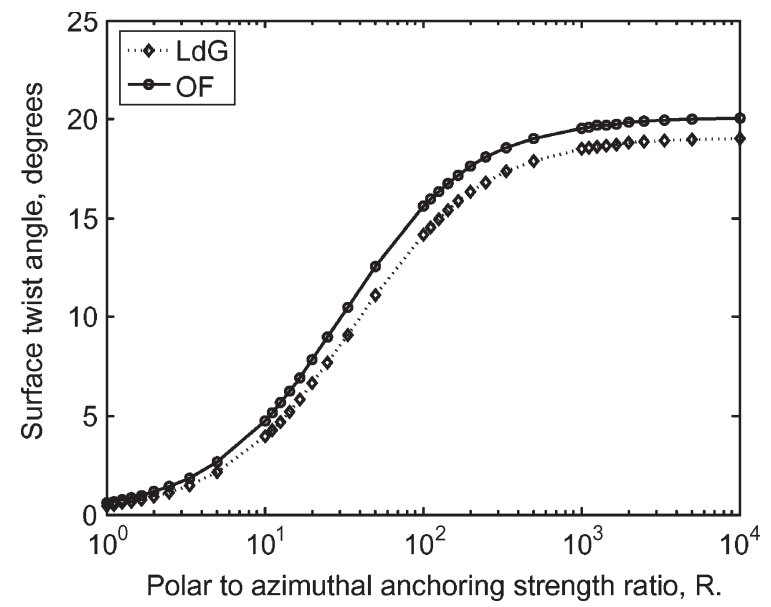

Fig. 12. Surface twist angles as a function of $R$.

taking into account the proportionality factor of $3 S_{e} / 2$, the effective azimuthal anchoring strength can be written as

$$
W_{1 \mathrm{eff}}=\frac{2 K_{22} \phi_{t}}{3 S_{0} d \sin (2 \Delta \phi)}
$$

where $\phi_{t}$ is the total twist angle in the LC material throughout the cell and, $d$ is the cell thickness.

Similarly, in the case of a planar cell (no twist), with equal but opposite pretilt on both surfaces and no applied voltage, the variation of the tilt angle across the cell can be assumed uniform. The effective polar anchoring strength $W_{2 \text { eff }}$ can be estimated using the Oseen-Frank theory with a one elastic constant approximation, giving

$$
W_{2 \mathrm{eff}}=\frac{2 K \theta_{t}}{3 S_{0} d \sin (2 \Delta \theta)}
$$

in terms of $\theta_{t}$, the total polar distortion angle through the cell, and $\Delta \theta$, the polar deviation angle of the director from the easy direction at the surface.

For both cells, starting with values of $W_{1}$ and $W_{2}$, the distribution of $\mathbf{Q}$ over the complete cell can be found by modeling using the Landau-de Gennes theory [28]. Then, using (24) and (25), the effective anchoring strength coefficients can be found from the tensor distribution. The ratio between $W_{i \text { eff }}$ and $W_{i}$ is plotted in Fig. 6 for a 1- $\mu$ m-thick cell with $K_{22}=K=$ $7 \mathrm{pN}$. The azimuthal and polar anchoring strengths were set as $W_{1}=85 \times 10^{-5} \mathrm{~J} / \mathrm{m}^{2}, W_{2}=W_{1} / R$ for $R>1$ and $W_{2}=$ $85 \times 10^{-5} \mathrm{~J} / \mathrm{m}^{2}, W_{1}=W_{2} R$ for $R<1$. When $R$ is close to 1 (almost uniaxial $\mathbf{Q}$ ), a good agreement between $W_{i}$ and $W_{i \text { eff }}$ is found. As $R$ departs from 1, the effective anchoring strength in the plane of increased biaxial order is reduced, whereas anchoring to the same plane is increased. That is, when $R<1$, $W_{\text {eff }}<W_{2}$ and when $R>1, W_{1 \text { eff }}<W_{1}$. The azimuthal anchoring strength shows a maximum change of approximately $7 \%$. It should be noted, however, that the difference between $W_{i \text { eff }}$ and $W_{i}$ depends on the degree of biaxiality at the surface and order parameter variation, so that the effective anchoring strength is a function of both bulk and surface terms.

\section{Discussion AND CONCLUSION}

A power series expansion in terms of $\mathbf{Q}$ and two mutually orthogonal unit vectors has been used to describe the anchoring energy density at the interface between a solid surface and a LC in the Landau-de Gennes theory. This expression allows for practical and flexible modeling of various weak anchoring types, ranging from isotropic through anisotropic to degenerate anchoring.

The lower order terms of the expansion have been considered, resulting in a simple expression with three coefficients. This allows the assignment of numerical values with a physical meaning to the scalar coefficients of the expression. Both the polar and azimuthal anchoring strengths can be independently defined, as well as the value of the easy surface-order parameter. Inclusion of higher order terms in $S$ and $P$ may allow for an improved description of variations in order, but this would introduce the disadvantage of added coefficients (material parameters) whose values need to be known. Furthermore, the low-order expansion simplifies in the constant uniaxial order limit to the well-known anisotropic generalization of the RP anchoring expression of Zhao et al. [15], [16].

Results of numerical simulations of the switching characteristics of a twisted test cell under various anchoring conditions and applied electric fields, using a finite element discretization of (10) in the Landau-de Gennes theory, compare well with those from the Oseen-Frank model. The resultant tilt and twist angles differ typically by less than $2^{\circ}$, and this can be explained by the fact that biaxiality and order variations are not considered in the Oseen-Frank formulation. It is also shown that as the anisotropy of the surface anchoring is increased, the surface order drops and becomes biaxial, resulting in an increase in effective anchoring strength to the plane of biaxial order and a decrease in anchoring strength in the same plane.

\section{REFERENCES}

[1] C. Oseen, "The theory of liquid crystals," Trans. Faraday Soc., vol. 29, pp. 883-889, 1933.

[2] F. C. Frank, "On the theory of liquid crystals," Discuss. Faraday Soc., vol. 29 , pp. $883-889,1933$

[3] P. G. de Gennes and J. Prost, The Physics of Liquid Crystals. Oxford, U.K.: Clarendon, 1993

[4] S. Ishihara, "How far has the molecular alignment of liquid crystals been elucidated?" J. Display Technol., vol. 1, no. 1, pp. 30-40, Sep. 2005. 
[5] A. V. Zakharov and R. Y. Dong, "Surface polarization and effective anchoring energy in liquid crystals," Phys. Rev. E, Stat. Phys. Plasmas Fluids Relat. Interdiscip. Top., vol. 64, no. 4, p. 042 701, Oct. 2001.

[6] A. V. Zakharov and M. Iwamoto, "Homeotropic-planar anchoring transition induced by trans-cis isomerization in ultrathin polyimide Langmuir-Blodgett films," J. Chem. Phys., vol. 118, no. 23, pp. $10758-$ 10761, Jun. 2003

[7] G. Barbero, I. Dozov, J. F. Palierne, and G. Durand, "Order electricity and surface orientation in nematic liquid crystals," Phys. Rev. Lett., vol. 56, no. 19, pp. 2056-2059, May 1986.

[8] A. L. Alexe-Ionescu, G. Barbero, and A. G. Petrov, "Gradient flexoelectric effect and thickness dependence of anchoring energy," Phys. Rev. E, Stat. Phys. Plasmas Fluids Relat. Interdiscip. Top., vol. 48, no. 3, pp. R1631R1634, Sep. 1993.

[9] A. Rapini and M. Papoular, "Distortion d'une lamelle nématique sous champ magnétique conditions d'ancrage aux parois," J. Phys. (Paris), vol. 30, no. C4.54, 1969.

[10] B. Zhang, P. Sheng, and H. S. Kwok, "Optical measurement of azimuthal anchoring strength in nematic liquid crystals," Phys. Rev. E, Stat. Phys. Plasmas Fluids Relat. Interdiscip. Top., vol. 67, no. 4, p. 041713 , Apr. 2003.

[11] S. Oka, T. Mitsumoto, M. Kimura, and T. Akahane, "Relationship between surface order and surface azimuthal anchoring strength of nematic liquid crystals," Phys. Rev. E, Stat. Phys. Plasmas Fluids Relat. Interdiscip. Top., vol. 69, no. 6, p. 061 711, Jun. 2004.

[12] A. Murauski, V. Chigrinov, A. Muravsky, F. S.-Y. Yeung, J. Ho, and S. Kwok, "Determination of liquid-crystal polar anchoring energy by electrical measurements," Phys. Rev. E, Stat. Phys. Plasmas Fluids Relat. Interdiscip. Top., vol. 71, no. 6, p. 061 707, Jun. 2005.

[13] T. Oh-Ide, S. Kuniyasu, and S. Kobayashi, "Surface coupling between nematic liquid crystals and rubbed polyimide substrates for pure twist deformation: Dependence on rubbing strength," Mol. Cryst. Liq. Cryst., vol. 164, no. 1, pp. 91-100, 1988

[14] B. Jérôme, "Surface effects and anchoring in liquid crystals," Rep. Prog. Phys., vol. 54, no. 3, pp. 391-451, Mar. 1991.

[15] W. Zhao, C.-X. Wu, and M. Iwamoto, "Analysis of weak-anchoring effect in nematic liquid crystals," Phys. Rev. E, Stat. Phys. Plasmas Fluids Relat. Interdiscip. Top., vol. 62, no. 2, pp. 1481-1484, Aug. 2000

[16] W. Zhao, C.-X. Wu, and M. Iwamoto, "Weak boundary anchoring, twisted nematic effect, and homeotropic to twisted-planar transition," Phys. Rev. E, Stat. Phys. Plasmas Fluids Relat. Interdiscip. Top., vol. 65, no. 3, p. 031709 , Mar. 2002

[17] R. Hirning, W. Funk, J.-R. Trebin, M. Schidt, and H. Schmiedel, "Threshold behavior and electro-optical properties of twisted nematic layers with weak anchoring in the tilt and twist angle," J. Appl. Phys., vol. 70, no. 8, p. 4211, Oct. 1991.

[18] J. Nehring, A. R. Kmetz, and T. J. Scheffer, "Analysis of weak-boundarycoupling effects in liquid-crystal displays," J. Appl. Phys., vol. 47, no. 3, p. 850, Mar. 1976.

[19] T. J. Sluckin and A. Poniewierski, "Novel surface phase transition in nematic liquid crystals: Wetting and the Kosterlitz-Thouless transition," Phys. Rev. Lett., vol. 55, no. 26, pp. 2907-2910, Dec. 1985.

[20] A. K. Sen and D. E. Sullivan, "Landau-de gennes theory of wetting and orientational transitions at a nematic-liquid-substrate interface," Phys. Rev. A, Gen. Phys., vol. 35, no. 3, pp. 1391-1403, Feb. 1987.

[21] M. Nobili and G. Durand, "Disorientation-induced disordering at a nematic-liquid-crystal-solid interface," Phys. Rev. A, Gen. Phys., vol. 46, no. 10, pp. R6174-R6177, Nov. 1992.

[22] A. Poniewierski and A. Samborski, "Anchoring of a nematic liquid crystal on an anisotropic substrate," Liq. Cryst., vol. 27, no. 10, pp. 1285-1293, Oct. 2000

[23] T. Akahane, H. Kaneko, and M. Kimura, "Novel method of measuring surface torsional anchoring strength of nematic liquid crystals," Jpn. J. Appl. Phys., vol. 35, no. 8, pp. 4434-4437, Aug. 1996.

[24] K. Okubo, M. Kimura, and T. Akahane, "Measurement of genuine azimuthal anchoring energy in consideration of liquid crystal molecular adsorption on alignment film," Jpn. J. Appl. Phys., vol. 42, no. 10, pp. 64286433, Oct. 2003.

[25] P. I. C. Teixeira, T. J. Sluckin, and D. E. Sullivan, "Erratum -Landau-de Gennes theory of anchoring transitions at a nematic liquid crystalsubstrate interface," Liq. Cryst., vol. 15, no. 6, p. 939, 1993.

[26] P. I. C. Teixeira, T. J. Sluckin, and D. E. Sullivan, "Landau-de Gennes theory of anchoring transitions at a nematic liquid crystal-substrate interface," Liq. Cryst., vol. 14, no. 4, pp. 1243-1253, 1993.

[27] H. J. Coles, "Laser and electric field induced birefringence studies on the cyanobiphenyl homologues," Mol. Cryst. Liq. Cryst., vol. 49, no. 1, pp. $67-74,1978$.
[28] R. James, E. Willman, F. A. Fernández, and S. E. Day, "Finite-element modelling of liquid crystal hydrodynamics with a variable degree of order," IEEE Trans. Electron Devices, vol. 53, no. 7, pp. 1575-1582, Jul. 2006.

[29] F. A. Fernández, S. E. Day, P. Trwoga, H. F. Deng, and R. James, "Three-dimensional modelling of liquid crystal display cells using finite elements," Mol. Cryst. Liq. Cryst., vol. 375, no. 1, pp. 291-299, 2002.

[30] S. Dickmann, J. Eschler, O. Cossalter, and D. A. Mlynski, "A simulation of LCDs including elastic anisotropy and inhomogeneous fields," in Proc. SID Dig., 1993, p. 638.

[31] H. Mori, J. E. C. Gartland, J. R. Kelly, and P. Boss, "Multidimensional director modelling using the $Q$ tensor representation in a liquid crystal cell and its application to the $\pi$ cell with patterned electrodes," Jpn. J. Appl. Phys., vol. 38, no. 1A, pp. 135-146, Jan. 1999.

[32] P. P. Karat and N. V. Madhusudana, "Elasticity and orientational order in some 4'-n-alkyl-4-cyanobiphenyls: Part II," Mol. Cryst. Liq. Cryst., vol. 40, no. 1, pp. 239-245, 1977.

[33] P. G. Cummings, D. A. Dunmur, and D. A. Laidler, "Dielectric properties of nematic 44'n-pentylcyanobiphenyl," Mol. Cryst. Liq. Cryst., vol. 30, no. 1/2, pp. 109-123, 1975.

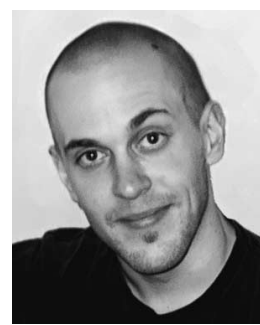

Eero Willman received the M.Eng. degree in electronic and electrical engineering from the Department of Electronic and Electrical Engineering, University College London, London, U.K., in 2003, where he is currently working toward the Ph.D. degree.

His current research interests include computer modeling and development of modeling tools for the simulation of nematic-liquid-crystal devices.

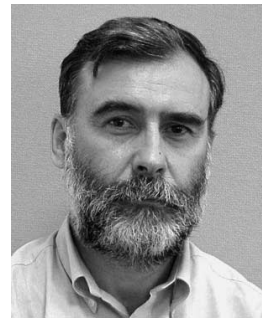

F. Aníbal Fernández (M'88) received the B.Sc. degree in applied mathematics from the Universidad de Chile, Santiago, Chile, in 1969, and the Ph.D. degree in electrical engineering from the University College London, London, U.K., in 1981.

Until 1985, he was on the academic staff with the Department of Electrical Engineering, Universidad de Chile. In 1986, he was with the staff of the Department of Electronic and Electrical Engineering, University College London, where he is currently a Senior Lecturer. His research interests include microwave and optical aspects of electromagnetic theory, particularly the use of numerical methods in those fields and, in the last ten years, have concentrated mainly in the modeling of liquid-crystal devices.

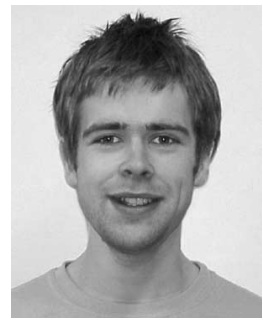

Richard James received the M.Eng. and Ph.D. degrees in electronic and electrical engineering from the University College London, London, U.K., in 2001 and 2006, respectively.

$\mathrm{He}$ is currently working as a Research Fellow in the LC modeling group at the same institution. His research interests include modeling of LC devices for displays and communications.

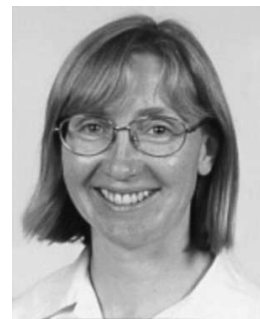

Sally E. Day (M'00) received the degree in physics from St. Hilda's College, Oxford University, Oxford, England, and the D.Phil. degree from Oxford University in 1988.

She spent five years with Thorn EMI, Central Research Laboratories, and Royal Signals and Radar Establishment, Malvern, studying various aspects of the optical properties of LCs, including the nonlinear optical properties. She is currently a Senior Lecturer with the Electronic and Electrical Engineering Department, University College London. Her research interests are the application of LCs in displays and nondisplay devices.

Dr. Day received the Royal Society University Research Fellowship held in the Electronic and Electrical Engineering Department, University College London in 1992. 